\title{
Editorials
}

\section{Faecal immunochemical testing in general practice}

The detection of invisible blood in faeces to diagnose colorectal cancer (CRC) has evolved with the introduction of the faecal immunochemical test - commonly referred to as FIT. It will soon replace the guaiacbased faecal occult blood test (gFOBT) in the NHS Bowel Cancer Screening Programme and has been recommended by the National Institute for Health and Care Excellence (NICE) for use in low-risk symptomatic patients ${ }^{1}$ with suspected CRC. There is tremendous enthusiasm to introduce FIT into 2-week wait (2WW) pathways to reduce referrals of patients without significant bowel disease and improve patient experience, free up overstretched endoscopy capacity, ${ }^{2}$ and save costs. But FIT is not without its shortcomings, and GPs will need to be aware of the limitations of this test, in addition to its exciting potential.

\section{FIT BENEFITS}

The clear and overriding benefit of FIT is its precision to detect the degradation products of blood in faeces, measured in $\mu \mathrm{g}$ of blood per gram of faeces $(\mu \mathrm{g} / \mathrm{g})$. Consequently, its sensitivity for CRC is significantly better than gFOBT in NICE modelling, at $92-100 \%$ vs 50\% respectively. ${ }^{3-5}$ FIT carries other advantages over gFOBT (Box 1), most notably its greater accuracy in detection of CRC in symptomatic patients. ${ }^{6,7}$ Although its sensitivity and specificity are excellent, nonetheless there are patients who will have false-positive and false-negative results with FIT, leading respectively to unnecessary investigation or missed cancers.

\section{HOW DOES FIT 'FIT' INTO NICE GUIDELINES}

In July 2017, NICE released its diagnostic guidance (DG30), which recommended the use of FIT in low-risk patients with suspected CRC, based on evidence evaluated in a health technology assessment (HTA). ${ }^{1}$ The sensitivity and specificity of FIT for CRC at a cut-off of $10 \mu \mathrm{g} / \mathrm{g}$ of blood in faeces was promising, but the HTA acknowledged several limitations to the data in the studies evaluated. Chief among these was the absence of data on patients with 'low-risk' symptoms. Instead, data were taken from studies that involved 'all comers' including high-risk symptoms in patients referred to secondary care for investigation. The only data on FIT from England was published as a conference abstract. The review acknowledged that there was variation in optimal thresholds for FIT in different countries and therefore data from studies outside of England may not be applicable to an English population. ${ }^{8}$

Despite the lack of evidence, the HTA and DG30 committee concluded that FIT could be used in low-risk patients with a cut-off of $10 \mu \mathrm{g} / \mathrm{g}$ but recommended that further largescale studies were needed to fully evaluate the diagnostic accuracy of FIT in low-risk patients. Without this research, implementation of FIT across the board in all low-risk symptomatic patients may lead to an endoscopy capacity crisis because of patients with false-positive FIT results, but more seriously may lead to delayed diagnosis for patients with falsenegative FIT results.

\section{FALSE-POSITIVE FIT}

DG30 recommended that FIT is performed in patients without rectal bleeding who have unexplained symptoms but do not meet the criteria for a suspected cancer. ${ }^{3}$ Literal interpretation of the guidance will lead to the eligibility of patients with any bowel or abdominal symptom (in the absence of rectal bleedingl, of any age, who do not meet 2WW criteria. Previous data reported in 2007 estimated that approximately $10 \%$ of primary care consultations were for gastrointestinal symptoms. ${ }^{9.10}$ The pool of eligible patients with gastrointestinal symptoms is therefore unquantified, but possibly enormous. Referral of these patients to secondary care would overwhelm endoscopy services, and many patients would undergo unnecessary investigation.

This potential situation was anticipated in the previous NICE 2015 (NG12) guidelines, which first established specific referral criteria for low-risk patients onto the 2-week pathway (Box 2). To avoid a deluge of referrals, NG12 recommended that only patients with a positive gFOBT from this group were referred for further investigations.

FIT has now supplanted gFOBT in the pathway for symptomatic patients. At 76.6$85.8 \%{ }^{1}$ 'the specificity of FIT at a cut-off of 10 is relatively high but this means that up to $25 \%$ of patients will have a false-positive result. As the broader DG30 criteria create a potentially enormous pool of low-risk patients, triage with FIT may lead to an overall increase in patients with false-positive results, and a higher number of referrals for further investigation. Limiting this pool of patients to specific symptom criteria (for example, the same low-risk criteria for gFOBT in NG12 guidance [Box 2], also recommended by CRUK) would prevent a surge in referrals.

\section{FALSE-NEGATIVE FIT}

Although false-positive referrals from DG30 have worrying implications for endoscopy service provision and costs, false-negative results will have the more devastating clinical impact on patients.

The sensitivity of FIT is very high, but not $100 \%:^{7}$ up to $10 \%$ of patients with CRC will have a false-negative FIT result. These patients may then be falsely reassured, and present only after their symptoms significantly worsen at a later date with advanced disease. This may be particularly true for low-risk patients, where symptoms may be reasonably attributed to other more likely causes such as IBS or haemorrhoids. As GPs, gastroenterologists, and colorectal surgeons will all be unfamiliar with FIT, safety netting will have a critical role to play in the introduction of this new healthcare technology. However, these pathways have not been established.

Work in the last year or so is gradually accumulating, highlighting the limitations of

\begin{tabular}{|c|c|}
\hline \multicolumn{2}{|c|}{ Box 1. gFOBT versus FIT comparison } \\
\hline gFOBT & FIT \\
\hline Sensitivity for CRC: $50.0 \%$ (15.0\% to $85.0 \%)$ & $\begin{array}{l}\text { Sensitivity for CRC (cut-off of } 10 \mu \mathrm{gg} / \mathrm{g} \text { ): } \\
92.1 \%(95 \% \mathrm{Cl}=86.9 \% \text { to } 95.3 \%)\end{array}$ \\
\hline Specificity for CRC: $88.0 \%$ (85.0\% to $89.0 \%$ ) & $\begin{array}{l}\text { Specificity for CRC (cut-off of } 10 \mathrm{ug} / \mathrm{g} \text { ): } \\
85.8 \%(95 \% \mathrm{Cl}=78.3 \% \text { to } 91.0 \%)\end{array}$ \\
\hline Qualitative & $\begin{array}{l}\text { Quantitative: cut-offs can be adjusted, altering test } \\
\text { sensitivity and specificity }\end{array}$ \\
\hline 2 samples from 3 stools & 1 sample from 1 stool \\
\hline $\begin{array}{l}\text { Dietary interference: false positives (meat), false } \\
\text { negatives (vitamin C) }\end{array}$ & No dietary interference \\
\hline False positives from upper GI bleeding & No false positives from upper GI bleeding \\
\hline
\end{tabular}




\section{Box 2. NG12 versus DG30 symptom criteria for $g F O B T / F I T$}

\section{NG12 gFOBT criteria}

- Aged $\geq 50$ years with abdominal pain or weight loss

- Aged $<60$ years with changes in bowel habit or iron deficiency anaemia

- Aged $>60$ years and with anaemia lin absence of iron deficiency)

FIT = faecal immunochemical test. $g F O B T=$ guaiac-based faecal occult blood tes

FIT, including possible contraindications to its use and under what conditions. Evidence from Scotland and Nottingham in symptomatic patients has suggested that patients with iron deficiency anaemia may have falsenegative FIT results. Initial work in Guildford, yet unpublished, has suggested that this may also be true for patients with the beta thalassaemia trait, whose globin-variant is not detected by the current FIT immunoassay. Research in London and Edinburgh is evaluating the role of other biomarkers such as circulating tumour DNA and methylated DNA in augmenting FIT sensitivity. This and similar research to optimise sensitivity of FIT is very important to avoid missing CRC

\section{WHERE WE ARE NOW AND THE SUGGESTED WAY FORWARD}

There is no doubt that FIT will revolutionise the pathway for all symptomatic patients with suspected CRC. At present, it has been recommended for low-risk patients, but it could potentially be used for all symptomatic patients once an evidence base has been established. This evidence is currently being gathered through large-scale cohort studies conducted through Croydon and Royal Marsden Partners, University College Collaborative, and York. The results are likely to be amalgamated and analysed by NHS England before rolling out the test nationally. FIT is currently being rolled out for low-risk patients as per DG30 guidance. It has also already been introduced for medium- to highrisk patients in Scotland, and more recently in Nottingham and Leicester. The results of safety-netting pathways and outcomes of patients with false-negative results will be informative but require time to accumulate enough data before analysis.

CCGs will need to consider the risks and benefits of FIT implementation for low-risk patients, which remain unknown in England. If FIT is introduced now, it may give GPs the opportunity to manage highly anxious low-risk patients and pick up some early cancers otherwise undetected. It may also lead to reduced referrals for investigation, less unnecessary tests for patients without significant bowel disease, improved patient

\section{DG30 FIT criteria}

- Patients without rectal bleeding who have unexplained symptoms but do not meet the criteria for a suspected cancer

\section{ADDRESS FOR CORRESPONDENCE}

\section{Nigel D'Souza}

Department of Colorectal Surgery, Croydon

University Hospital, Croydon CR7 7YE, UK.

\section{Email: nigel.d'souzaßanhs.net}

atherealnige safety and experience, a reduction in overstretched endoscopy utilisation, and cost savings for the NHS. Patients with falsenegative results may be safety netted adequately, and avoid delayed presentation. But, conversely, the opposite may happen. To avoid this situation, FIT could be used only in low-risk patients meeting the previous symptom criteria for gFOBT in the NICE NG12 guidance; this is an approach supported by CRUK

While considering this, CCGs may want to bear in mind that triage for referral of lowrisk symptomatic patients has been ongoing for some time using a more old-fashioned 'technology': clinical judgement of GPs. The CRC stage of symptomatic patients referred urgently by GPs that do not meet 2WW criteria is equivalent to patients referred automatically with 2WW based on symptoms." Because GP triage performance is equivalent to $2 \mathrm{WW}$ criteria, FIT implementation for all low-risk symptomatic patients could be delayed without detriment to patient outcomes, until research findings and safety-netting outcomes have been analysed by NHS England.

\section{Nigel D'Souza,}

Colorectal Research Fellow, Department of Colorectal Surgery, Croydon University Hospital, Croydon.

\section{Anthony Brzezicki,}

Clinical Lead for Cancer, Healthy London Partnership Cancer Programme, London.

\section{Muti Abulafi,}

Consultant Colorectal Surgeon, Croydon University Hospital, Croydon.

\section{Provenance}

Commissioned; externally peer reviewed.

\section{Competing interests}

Nigel D'Souza and Muti Abulafi are investigators for the NICE FIT Study.

DOI: https://doi.org/10.3399/bjgp19X700853

\section{REFERENCES}

1. Westwood M, Corro Ramos I, Lang S, et al. Faecal immunochemical tests to triage patients with lower abdominal symptoms for suspected colorectal cancer referrals in primary care: a systematic review and cost-effectiveness analysis. Health Technol Assess 2017; 21(33): $1-234$

2. Shenbagaraj L, Thomas-Gibson S, Stebbing J, et al. Endoscopy in 2017: a national survey of practice in the UK. Frontline Gastroenterol 2018: 10(1): $7-15$

3. National Institute for Health and Care Excellence. Quantitative faecal immunochemical tests to guide referral for colorectal cancer in primary care. DG30. London: NICE, 2017. https://www.nice.org.uk/guidance/dg30 laccessed 13 Dec 2018).

4. National Institute for Health and Care Excellence. Suspected cancer: recognition and referral. NG12. London: NICE, 2015. https:// unw.nice.org.uk/guidance/ng12 laccessed 13 Dec 2018).

5. Gillberg A, Ericsson E, Granstrom F, Olsson L A population-based audit of the clinical use of faecal occult blood testing in primary care for colorectal cancer. Colorectal Dis 2012; 14(9): e539-e546.

6. Steele RJC. Fraser CG. Faecal immunochemical tests (FIT) for haemoglobin for timely assessment of patients with symptoms of colorectal disease. In: Olsson L, ed. Timely diagnosis of colorectal cancer. Cham: Springer International Publishing AG, 2018: 39-66.

7. Fraser $C G$. Faecal immunochemical tests (FIT) in the assessment of patients presenting with lower bowel symptoms: concepts and challenges. Surgeon 2018; 16(5): 302-308.

8. Fraser CG, Rubeca T, Rapi S, et al. Faecal haemoglobin concentrations vary with sex and age, but data are not transferable across geography for colorectal cancer screening. Clin Chem Lab Med 2014; 52(8): 1211-1216.

9. Williams JG, Roberts SE, Ali MF, et al. Gastroenterology services in the UK. The burden of disease, and the organisation and delivery of services for gastrointestinal and liver disorders: a review of the evidence. Gut 2007; 56(Suppl 1): $1-113$

10. Jones R, Hunt C, Stevens R, et al. Management of common gastrointestinal disorders: quality criteria based on patients' views and practice guidelines. Br J Gen Pract 2009; DOI: https://doi. org/10.3399/bjgp09X420761

11. National Cancer Registration and Analysis Service. Routes to diagnosis by stage 2012-2013 workbook. 2015. http://uww.ncin.org.uk/ view?rid=3071 laccessed 14 Dec 2018) 